\title{
Evaluation of Sienna Cancer Diagnostics hTERT Antibody on 500 Consecutive Urinary Tract Specimens
}

\author{
Derek B. Allison ${ }^{\mathrm{a}}$ Rajni Sharma ${ }^{\mathrm{a}}$ Morgan L. Cowan $^{\mathrm{a}}$ \\ Christopher J. VandenBussche ${ }^{a, b}$ \\ ${ }^{a}$ Department of Pathology, Johns Hopkins University School of Medicine, Baltimore, MD, USA; ${ }^{b}$ Department of \\ Oncology, Johns Hopkins University School of Medicine, Baltimore, MD, USA
}

\section{Keywords \\ Urine cytology $\cdot$ Urinary tract $\cdot$ Telomerase $\cdot$ Ancillary test}

\begin{abstract}
Objectives: Telomerase activity can be detected in up to $90 \%$ of urothelial carcinomas (UC). Telomerase activity can also be detected in urinary tract cytology (UTC) specimens and indicate an increased risk of UC. We evaluated the performance of a commercially available antibody that putatively binds the telomerase reverse transcriptase (hTERT) subunit on 500 UTC specimens. Study Design: Unstained Cytospin ${ }^{\mathrm{TM}}$ preparations were created from residual urine specimens and were stained using the anti-hTERT antibody (SCDA7). Two algorithms were developed for concatenating the hTERT result and cytologic diagnosis: a "no indeterminates algorithm," in which a negative cytology and positive hTERT result are considered positive, and a "high-specificity algorithm," in which a negative cytology and positive hTERT result are considered indeterminate (and thus negative for comparison to the gold standard). Results: The "no indeterminates algorithm" and "high-specificity algorithm" yielded a sensitivity of 60.6 and $52.1 \%$, a specificity of 70.4 and $90.7 \%$, a positive predictive value of 39.1 and $63.8 \%$, and a negative
\end{abstract}

\begin{tabular}{ll}
\hline KARGER & ( ) 2018 The Author(s) \\
Published by S. Karger AG, Basel & Karger \\
E-Mail karger@karger.com & This article is licensed under the Creative Commons Attribution- \\
www.karger.com/acy & $\begin{array}{l}\text { NonCommercial-NoDerivatives 4.0 International License (CC BY- } \\
\text { NC-ND) (http://www.karger.com/Services/OpenAccessLicense). } \\
\text { Usage and distribution for commercial purposes as well as any dis- } \\
\text { tribution of modified material requires written permission. }\end{array}$
\end{tabular}

predictive value of 85.0 and $85.8 \%$, respectively. Conclusions: A positive hTERT result may identify a subset of patients with an increased risk of high-grade UC (HGUC) who may otherwise not be closely followed, while a negative hTERT immunocytochemistry result is associated with a reduction in risk for HGUC.

(C) 2018 The Author(s)

Published by S. Karger AG, Basel

\section{Introduction}

Urinary tract carcinoma (UC) is a treatable but burdensome disease that requires continual surveillance because of the high risk of recurrence $[1,2]$. The visualization of tumors during cystoscopy and ureteroscopy is considered the gold standard method of detection but is an invasive procedure that is time-consuming, costly, and uncomfortable for the patient. Urinary tract cytology (UTC) is a useful, noninvasive adjunct for surveillance because of its high specificity for the detection of highgrade urothelial carcinoma (HGUC) $[3,4]$. Unfortunately, UTC has limited sensitivity for the detection of HGUC

D.B.A. and R.S. contributed equally.
Correspondence to: Dr. Christopher J. VandenBussche Department of Pathology, Johns Hopkins Hospital Carnegie 406, 600 N. Wolfe Street

Baltimore, MD 21287 (USA)

E-Mail cjvand@jhmi.edu 
and poor sensitivity and specificity for the detection of low-grade urothelial neoplasms, such as low-grade urothelial carcinoma (LGUC) [5, 6]. A number of noninvasive, ancillary tests have been developed and reported in the literature $[7,8]$. Some, such as FISH, are used in conjunction with UTC, while others are used independently of UTC results; however, none of these tests have gained universal acceptance.

Studies have detected telomerase activity in up to $90 \%$ of UC. Furthermore, telomerase activity can be detected in UTC and indicates an increased risk for having UC [913]. hTERT (telomerase reverse transcriptase) is the catalytic subunit component of the telomerase ribonucleoprotein complex. The majority of UC (60-80\%) have mutations in the TERT promoter, which can also be found in some histologic variants of UC [14-18]. A small study of 101 cell blocks created from urinary sediment found that hTERT immunostaining had a sensitivity of $84.8 \%$ and specificity of $65.2 \%$ for the detection of UC [19].

In this study, we investigate the performance of a commercially available antibody that putatively binds hTERT. To do so, we performed immunocytochemistry (ICC) and blindly interpreted the result on 500 consecutive UTC specimens submitted to our laboratory.

\section{Materials and Methods}

\section{Specimen Cohort}

The institutional review board approved this study and provided a consent waiver. 500 consecutive residual urine specimens from 474 unique patients submitted to the cytopathology laboratory for clinical diagnosis were utilized, with specimens only being excluded if insufficient residual material to create an experimental preparation remained following the rendering of a clinical diagnosis. Specimens with a volume of $\geq 20 \mathrm{~mL}$ were stored at $2-8^{\circ} \mathrm{C}$ and subsequently processed in batches every 2-3 days.

Clinical specimens were prepared using a minimum of $25 \mathrm{~mL}$ of fresh urine and processed using the BD SurePath ${ }^{\circledR}$ liquid-based preparation. The clinical diagnosis was rendered by 1 of 5 cytopathology-boarded pathologists in the Johns Hopkins Hospital (JHH) Division of Cytopathology.

Diagnoses were made using the Johns Hopkins Template for Reporting Urinary Tract Cytology (JHHT) because the Paris System for Reporting Urinary Tract Cytology had not yet been established at JHH during this study period [20-22]. The JHHT utilized a malignant category, "high-grade urothelial carcinoma" (HGUC), a benign category, "no urothelial atypia or malignancy" (NUAM), a low-risk indeterminate category, "atypical urothelial cells of undetermined significance" (AUC-US), and a high-risk indeterminate category, “atypical urothelial cells, cannot exclude HGUC" (AUC-H).

\section{Experimental Slide Preparation}

Specimens were transferred and centrifuged in $50-\mathrm{mL}$ centrifuge tubes at $470 \mathrm{~g}$ for $10 \mathrm{~min}$ at $2-8^{\circ} \mathrm{C}$. The supernatant was dis- carded, and the cell pellet resuspended in $10 \mathrm{~mL}$ phosphate-buffered saline, $\mathrm{pH} 7.4$, prior to being centrifuged at $470 \mathrm{~g}$ for $10 \mathrm{~min}$ at $2-8{ }^{\circ} \mathrm{C}$. This step was repeated if the cell pellet was found to contain large proteinaceous material upon inspection. Cell pellets were then resuspended in $10 \mathrm{~mL}$ alcohol containing carbowax (PEG) fixative (Shandon ${ }^{\mathrm{TM}}$ Cytospin ${ }^{\mathrm{TM}}$ Collection Fluid, Thermo Scientific ${ }^{\mathrm{TM}}$ ) and centrifuged at $470 \mathrm{~g}$ for $10 \mathrm{~min}$ at $2-8^{\circ} \mathrm{C}$.

The supernatant was discarded, and fixed cell pellets were resuspended in a $500-\mu \mathrm{L}$ to $1-\mathrm{mL}$ fixative solution. Specimen cells are affixed to slides by adding the cell suspensions to a Cytospin ${ }^{\mathrm{TM}}$ funnel attached via a cytoclip to positively charged glass microscope slide and centrifuged at $113 \mathrm{~g}(1,000 \mathrm{rpm})$ for 4 min with a low acceleration setting using a Cytopsin ${ }^{\mathrm{TM}} 4$ Cytocentrifuge (Thermo Scientific ${ }^{\mathrm{TM}}$ ). Three slides were prepared using the thinlayer liquid-based cytology preparation for each patient specimen. Slides were subsequently stored and held in slide boxes at $2-8^{\circ} \mathrm{C}$ until ICC was carried out.

\section{Immunocytochemistry}

Anti-hTERT antibody (SCD-A7) is a mouse monoclonal antibody produced as a tissue culture supernatant and supplied in phosphate-buffered saline with carrier protein, containing $0.05 \%$ ProClin ${ }^{\circledR} 300$ as a preservative (Sienna Cancer Diagnostics Ltd., Melbourne, Australia, used at $5.4-6.6 \mu \mathrm{g} / \mathrm{mL}$ ).

Slides were removed from the $2-8^{\circ} \mathrm{C}$ storage and placed onto the Ventana BenchMark Ultra automated staining platform (Ventana Medical Systems Inc., Tucson, AZ, USA). Slides were treated with heat-induced epitope retrieval performed as follows: Ultra Cell Conditioning (Ultra CC1, $\mathrm{pH} 8.5$ ) retrieval buffer for $8 \mathrm{~min}$ at $95^{\circ} \mathrm{C}$. Slides were then incubated with the diluted (1/11) antihTERT antibody for $44 \mathrm{~min}$ at $37^{\circ} \mathrm{C}$. Nonspecific antibody binding was blocked by incubating cytology specimens with OptiView peroxidase inhibitor containing $3 \%$ hydrogen peroxidase solution and DISCOVERY antibody diluent (Ventana Medical Systems Inc., Tucson, AZ, USA) for $8 \mathrm{~min}$. For hTERT immunocytochemical staining, the OptiView DAB IHC detection kit (Ventana Medical Systems Inc., Tucson, AZ, USA) was used which makes use of a cocktail of secondary antibodies that locate the bound primary antibody. The slides were incubated with OptiView HQ universal linker and OptiView HRP multimer for $8 \mathrm{~min}$ at $37^{\circ} \mathrm{C}$. The secondary antibody cocktail was recognized by an enzyme-bound, tertiary antibody that was visualized with hydrogen peroxide substrate and the 3,3'-diaminobenzidine tetrahydrochloride chromogen, which produces a brown precipitate that is readily detected by light microscopy. Slides were counterstained with hematoxylin II (Ventana Medical Systems Inc., Tucson, AZ, USA) and then mounted.

\section{Experimental Slide Interpretation}

Interpretations were performed by 1 board-certified cytopathologist (C.J.V.). The interpretation of hTERT ICC was determined based on preliminary observations made during previous antibody optimization runs and recommendations made by Sienna Cancer Diagnostics, which were finalized prior to the blind reading of the experimental slides and are described below.

The cutoff for determining a positive hTERT ICC result was nuclear staining of: (1) at least 1 urothelial cell with atypical cytomorphology or (2) at least 2 cytomorphologically benign-appearing urothelial cells. Cytoplasmic staining was not regarded as a positive result and its presence did not invalidate a positive nucle- 


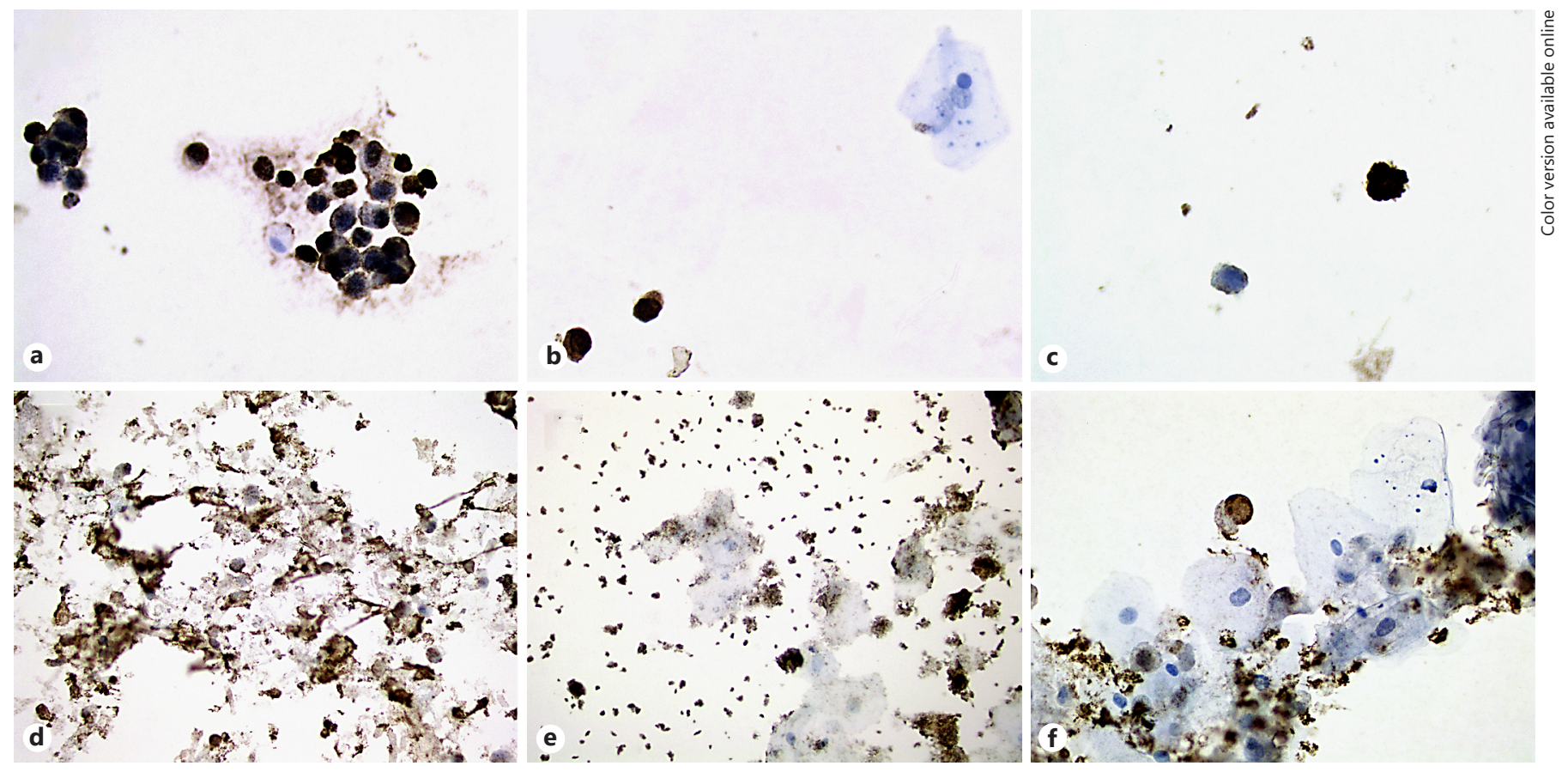

Fig. 1. Examples of hTERT interpretation. a Numerous malignant cells are seen, more than half demonstrating positive nuclear staining. The cells have recognizable and intact cytoplasm on the counterstain and are morphologically compatible with urothelial cells; thus, it is appropriate to interpret them as hTERT positive. The presence of cytoplasmic staining in some of these cells does not invalidate the result. b Two cells (lower left) have nuclear staining and recognizable, intact cytoplasm. The cells are morphologically compatible with urothelial cells; a mature squamous cell (upper right) acts as an internal negative control. c A cluster of bacteria stain positively by hTERT and approximate the size of a urothelial cell nucleus. The stain was not interpreted as positive if a cell's cytoplasm was not clearly seen. $\mathbf{d - f}$ Numerous positive-staining bacteria are present in the background, stuck to mature squamous cells. In cases where positive-staining inflammatory cells and/or bacteria obscured the preparation, the test was considered noninterpretable. $\mathbf{f}$ Despite positive-staining bacteria at the bottom of the field, a positive urothelial cell is clearly seen in the center of the field. In such instances, cases could be interpreted as positive. ar stain. The following nonurothelial components consistently stained positively: bacteria, lymphocytes, and neutrophils. Occasional nuclear staining of mature squamous cells could be identified and was not regarded as a positive result. Positive-staining granular debris of unknown origin was sometimes present in the background and was not considered a positive result. The hTERT ICC result was considered uninterpretable if: (1) less than 5 urothelial cells were present for evaluation or (2) any positive-staining nonurothelial component interfered with the interpretation of the urothelial cell component. Cells that could not be definitively determined to be of squamous versus urothelial origin were considered to be urothelial cells (Fig. 1).

\section{Gold Standard Comparison}

To compare the cytologic diagnoses and test interpretation against a gold standard result, we considered results from the patient's concurrent cystoscopy, concurrent and subsequent tissue biopsies, and subsequent UTC specimen results. The follow-up period ranged from 6 to- 12 months depending on the time at which the specimen was collected and the time at which the slides were stained and clinical data were obtained. Either a positive diagnosis for a urothelial carcinoma (HGUC or LGUC) on biopsy or a diagnosis of
HGUC with UTC were considered as a positive follow-up regardless of the cystoscopy result. In the absence of a follow-up biopsy or positive UTC specimen, a positive cystoscopy result was considered as a positive follow-up. A positive cystoscopy was considered negative if a follow-up biopsy occurred and was negative for UC.

\section{Results}

\section{Study Cohort}

The study cohort contained 410 specimens in which the test result was considered interpretable (Fig. 1). 297 (72.4\%) of these specimens had an associated concurrent cystoscopy or follow-up specimen (as defined in the Methods section) as a gold standard for comparison. Associated patient information is summarized in Table 1; because these specimens were de-identified, some specimens may represent repeat specimens from the same patient, which is not accounted for in the demographic data. 
Table 1. Characteristics of the 297 specimens with an interpretable hTERT immunocytochemistry result

\begin{tabular}{|c|c|}
\hline \multicolumn{2}{|l|}{ Age, years } \\
\hline Mean & 67.6 \\
\hline Range & 15-95 \\
\hline \multicolumn{2}{|l|}{ Race, $\%(n)$} \\
\hline White & $75.4(224)$ \\
\hline Black & $14.8(44)$ \\
\hline Other/unknown & $9.8(29)$ \\
\hline \multicolumn{2}{|l|}{ Sex, $\%(n)$} \\
\hline Male & $80.5(239)$ \\
\hline Female & $19.5(58)$ \\
\hline \multicolumn{2}{|l|}{ Specimen type, \% ( $n)$} \\
\hline Voided & $83.5(248)$ \\
\hline Catheterized & $8.1(24)$ \\
\hline Washing & $7.7(23)$ \\
\hline Unknown procurement method & $0.7(2)$ \\
\hline \multicolumn{2}{|l|}{ Cytologic diagnosis, $\%(n)$} \\
\hline NUAM & $64.0(190)$ \\
\hline AUC-US & $26.9(80)$ \\
\hline AUC-H & $5.4(16)$ \\
\hline HGUC & $3.7(11)$ \\
\hline \multicolumn{2}{|l|}{ Cystoscopy, \% (n) } \\
\hline Performed, negative & $24.9(74)$ \\
\hline Performed, positive & $67.0(199)$ \\
\hline Not performed & $7.4(22)$ \\
\hline Not reported & $0.7(2)$ \\
\hline \multicolumn{2}{|l|}{ Follow-up specimens, $\%(n)$} \\
\hline None & $29.6(88)$ \\
\hline Benign UTC specimens only & $37.0(110)$ \\
\hline HGUC on UTC & $1.7(5)$ \\
\hline Benign biopsy & $9.4(28)$ \\
\hline Atypical biopsy & $0.3(1)$ \\
\hline LGUC on biopsy & $8.0(24)$ \\
\hline HGUC on biopsy & $12.5(37)$ \\
\hline LGUC and HGUC on biopsy & $1.3(4)$ \\
\hline
\end{tabular}

HGUC, high-grade urothelial carcinoma; LGUC, low-grade urothelial carcinoma; UTC, urinary tract specimen; NUAM, negative for urothelial atypia or malignancy; AUC-US, atypical urothelial cells of undetermined significance; AUC-H, atypical urothelial cells, cannot exclude HGUC.

\section{Performance of Cytologic Diagnosis}

Among the cohort of 297 specimens with a gold standard for comparison, the distribution of initial cytology diagnoses was as follows: 190 NUAM, 80 AUC-US, 16 AUC-H, and 11 HGUC. Given our institution's high rate of HGUC among AUC-H diagnoses, NUAM and AUCUS diagnoses were considered negative, and HGUC and AUC-H were considered positive for comparison to the gold standard. The specificity of cytology within this test cohort for any urothelial carcinoma, regardless of grade, was $97.8 \%$ and the sensitivity was $31.0 \%$ (Fig. 2 ).

\section{Test Performance Using the "No Indeterminates" Algorithm}

In order to interpret the test result in the context of the cytologic result, 2 possible algorithms were applied. The "no indeterminates" algorithm interprets NUAM specimens with a positive hTERT result as an overall positive result (Table 2). When this algorithm was applied and the overall result compared to the gold standard, the specificity of the test was $70.4 \%$ and the sensitivity $60.6 \%$ (Fig. 3 , $4)$.

\section{Test Performance Using the "High-Specificity" \\ Algorithm}

The second algorithm ("high-specificity" algorithm) interprets NUAM specimens with a positive hTERT result as "atypical" and considered negative for comparison to the gold standard (Table 2). In this context, the specificity of the test was $90.7 \%$ and the sensitivity $52.1 \%$ (Fig. 5).

\section{Test Performance for LGUC}

The study cohort contained 24 specimens in which the patient was subsequently diagnosed with LGUC on tissue biopsy. The cytologic diagnosis was NUAM for 9 specimens and AUC-US for 15 specimens. Among these, only 3 specimens diagnosed as NUAM were hTERT ICC positive, and almost half (7/15) of those diagnosed as AUCUS were hTERT ICC positive. These results amount to a sensitivity of $41.6 \%$ according to the "no indeterminates" algorithm and a sensitivity of $29.2 \%$ according to the "high-specificity" algorithm.

\section{Predicting Test Impact in Instances Cystoscopy Was}

Not Performed or Negative

Assuming patients with positive cystoscopies would receive a closer follow-up regardless of cytologic diagnosis and those with HGUC or AUC-H diagnoses would receive a closer follow-up regardless of cystoscopy diagnosis, we investigated the impact of hTERT ICC results on those "lower-risk" patients who may not otherwise receive close follow-ups.

22 patients had no concurrent cystoscopy specimen; 3 were diagnosed with AUC-US and 19 with NUAM (22 total AUC-US or NUAM). 199 patients had negative concurrent cystoscopies; 46 were diagnosed with AUC-US, 143 with NUAM, 5 with AUC-H, and 5 with HGUC (189 total AUC-US or NUAM). Thus, 211 patients would not have been put into a higher risk category based on cytology and cystoscopy results alone. With hTERT test utilization in this subset of patients, 11 of the 211 would have 
Table 2. Final test interpretation for comparison to gold standard based on concatenation of urinary cytology specimen clinical diagnosis and hTERT result

\begin{tabular}{lll}
\hline Urine cytology result & hTERT ICC result & Overall urine result \\
\hline Atypical (AUC-US) & Negative & Negative \\
Atypical (AUC-US) & Positive & Positive \\
Negative (NUAM) & Negative & Negative \\
Positive (AUC-H or HGUC) & Positive & Positive \\
Positive (AUC-H or HGUC) & Negative & Positive \\
Negative & Positive & "Atypical" (negative) or positive \\
\hline
\end{tabular}

HGUC, high-grade urothelial carcinoma; NUAM, negative for urothelial atypia or malignancy; AUC-US, atypical urothelial cells of undetermined significance; AUC-H, atypical urothelial cells, cannot exclude HGUC. ${ }^{1}$ We present data from two algorithms, one in which a negative urine cytology result with a positive hTERT result combines to form an overall urine result that is considered "atypical" and thus negative for comparisons ("high-specificity" algorithm) to the gold standard. The second algorithm ("no indeterminates" algorithm) considers this combination to be positive for comparisons to the gold standard.

\begin{tabular}{|c|c|c|c|c|c|c|}
\hline \multicolumn{7}{|c|}{ Cytology alone } \\
\hline & & & & & \multicolumn{2}{|l|}{ Test statistics } \\
\hline & & \multicolumn{2}{|c|}{ Gold standard } & & Number & 297 \\
\hline & & Positive & Negative & & Sensitivity & $31.0 \%$ \\
\hline \multirow{2}{*}{ Urine cyto } & Positive & 22 & 5 & 27 & Specificity & $97.8 \%$ \\
\hline & Negative & 49 & 221 & 270 & Likelihood ratio for positive test $(\mathrm{LR}+)$ & 14.01 \\
\hline & & 71 & 226 & & Likelihood ratio for negative test (LR-) & 0.71 \\
\hline & & & & & Positive predictive value (PPV) & $81.5 \%$ \\
\hline & & & & & Negative predictive value (NPV) & $81.9 \%$ \\
\hline & & & & & Diagnostic accuracy & $81.8 \%$ \\
\hline
\end{tabular}

Fig. 2. Performance of cytology alone compared to the study's gold standard.

received an additional follow-up. Two of these 11 were ultimately diagnosed with HGUC (18.2\% prevalence compared to $2.8 \%$ prevalence in the larger subset). Of the remaining 200 patients lacking any positive result by hTERT ICC, cytology, and cystoscopy, 4 were ultimately diagnosed with HGUC (2.0\% prevalence compared to $2.8 \%$ prevalence in the larger subset). Therefore, while the test lacks some specificity for low-risk patients, a negative test is reassuring in this setting.

\section{Discussion}

Sensitivity for the test was significantly higher than cytology alone, especially in the ability to detect LGUC. The sensitivity of cytology alone is higher when low-grade lesions are not considered; this is an important tenant of the Paris System for Reporting Urinary Tract Cytology, given the extremely low sensitivity and specificity for detecting low-grade lesions by UTC. The additional detection of LGUC lesions contributed to 7 of 15 additional "true-positive" results by the "high-specificity" algorithm 
Fig. 3. Flowchart detailing the gold standard follow-up for each specimen, with associated combined hTERT and cytology results according to the "no indeterminates" and "high-specificity" algorithms.



and 10 of 21 additional true-positive results by the "no indeterminates" algorithm.

Concerning the biopsy-established LGUC cohort, a positive hTERT result correlated to the gold standard with a $42 \%$ sensitivity using the "no indeterminates" algorithm, or $29 \%$ using the "high-specificity" algorithm. This small difference is due to a positive hTERT ICC result in 2 specimens with a cytologic diagnosis of NUAM; the majority of hTERT ICC positive specimens with LGUC follow-up had cytologic diagnoses of AUC-US. Low-grade lesions sometimes contain recognizable atypia but have a decreased tendency to shed into the urine as compared to HGUC lesions [6]. The absence of cytologic atypia in the NUAM specimens may indicate that these specimens may not have contained any lesional cells and, thus, were more likely to be hTERT ICC negative. The majority $(9 / 10)$ of hTERT ICC positive specimens from patients with LGUC were voided urines. These findings suggest suggests that LGUC can shed into voided urine without requiring instrumentation for dislodgement and that the cytologic atypia associated with these lesions was not severe enough to result in a diagnosis of AUC-H or HGUC.

This current study's design inherently suffers due to the potential for "false-positives," in which a test may detect a lesion prior to the gold standard method [23]. Reports have shown this pitfall in other studies of UTC ancillary tests, and even positive cytomorphologic diagnoses may precede tissue biopsy confirmation by several months to years. This phenomenon is likely due to multiple factors, including the fact that cystoscopy at best has a sensitivity of $90 \%$, carcinoma in situ may be difficult to identify on cystoscopy, and dysplastic or early neoplastic changes may harbor detectable alterations. Some of the "false-positive" hTERT ICC results are instances in which cytology, hTERT ICC, and initial cystoscopy were all positive, yet the follow-up biopsy was negative for UC. In these circumstances, it is expected that some biopsies did not sample the relevant lesion, or that carcinoma in situ cells detached from the biopsy area, leaving only denuded tissue.

There were 9 specimens for which the "high-specificity" algorithm was positive, but the cystoscopy was initially reported as negative. Subsequent follow-up biopsy revealed HGUC or carcinoma in situ in 7 of those cases. This finding indicates that at least some of the "false-positive" hTERT ICC results correctly predicted either a higher likelihood for carcinoma (that was perhaps missed on initial cystoscopy) or for the development of carcinoma (identified on later cystoscopy). 


\begin{tabular}{|c|c|c|c|c|c|c|}
\hline \multicolumn{7}{|c|}{ No indeterminates algorithm } \\
\hline & & & & \multicolumn{2}{|l|}{ Test statistics } \\
\hline & & \multicolumn{2}{|c|}{ Gold standard } & & Number & 297 \\
\hline & & Positive & Negative & & Sensitivity & $60.6 \%$ \\
\hline \multirow{2}{*}{$\begin{array}{c}\text { Cyto } \\
+ \\
\text { hTERT }\end{array}$} & Positive & 43 & 67 & 110 & Specificity & $70.4 \%$ \\
\hline & Negative & 28 & 159 & 187 & Likelihood ratio for positive test (LR+) & 2.04 \\
\hline & & 71 & 226 & & Likelihood ratio for negative test (LR-) & 0.56 \\
\hline & & & & & Positive predictive value (PPV) & $39.1 \%$ \\
\hline & & & & & Negative predictive value (NPV) & $85 \%$ \\
\hline & & & & & Diagnostic accuracy & $68 \%$ \\
\hline
\end{tabular}

Fig. 4. Overall performance of cytology plus hTERT immunocytochemistry (ICC) compared to the study's gold standard. The "no indeterminates" algorithm considers a benign cytology specimen with a positive hTERT ICC result as an overall positive result for comparison to the gold standard.

\begin{tabular}{|c|c|c|c|c|c|c|}
\hline \multicolumn{7}{|c|}{ High-specificity algorithm } \\
\hline & & & & \multicolumn{2}{|l|}{ Test statistics } \\
\hline & & \multicolumn{2}{|c|}{ Gold standard } & & Number & 297 \\
\hline & & Positive & Negative & & Sensitivity & $52.1 \%$ \\
\hline \multirow{2}{*}{$\begin{array}{c}\text { Cyto } \\
+ \\
\text { hTERT }\end{array}$} & Positive & 37 & 21 & 58 & Specificity & $90.7 \%$ \\
\hline & Negative & 34 & 105 & 239 & Likelihood ratio for positive test $(\mathrm{LR}+)$ & 5.61 \\
\hline & & 71 & 226 & & Likelihood ratio for negative test (LR-) & 0.53 \\
\hline & & & & & Positive predictive value (PPV) & $63.8 \%$ \\
\hline & & & & & Negative predictive value (NPV) & $85.8 \%$ \\
\hline & & & & & Diagnostic accuracy & $81.5 \%$ \\
\hline
\end{tabular}

Fig. 5. Overall performance of cytology plus hTERT immunocytochemistry (ICC) compared to the study's gold standard. The "high-specificity" algorithm considers a benign cytology specimen with a positive hTERT ICC result as indeterminate and thus as an overall negative result when compared to the gold standard.

Regarding the hTERT ICC test, there are 2 limitations that prevent higher levels of sensitivity from being achieved. The first is that with exfoliative UTC specimens, the ability to detect cancer depends on those cells having been shed into the urine at the time of sample collection. Secondly, a small proportion of UC do not have alterations in telomerase activity. Indeed, in the current study, 2 of 11 specimens with a cytologic diagnosis of HGUC were hTERT ICC negative, which corresponds to reports that telomerase activity is detectable in up to $90 \%$ of UC. 


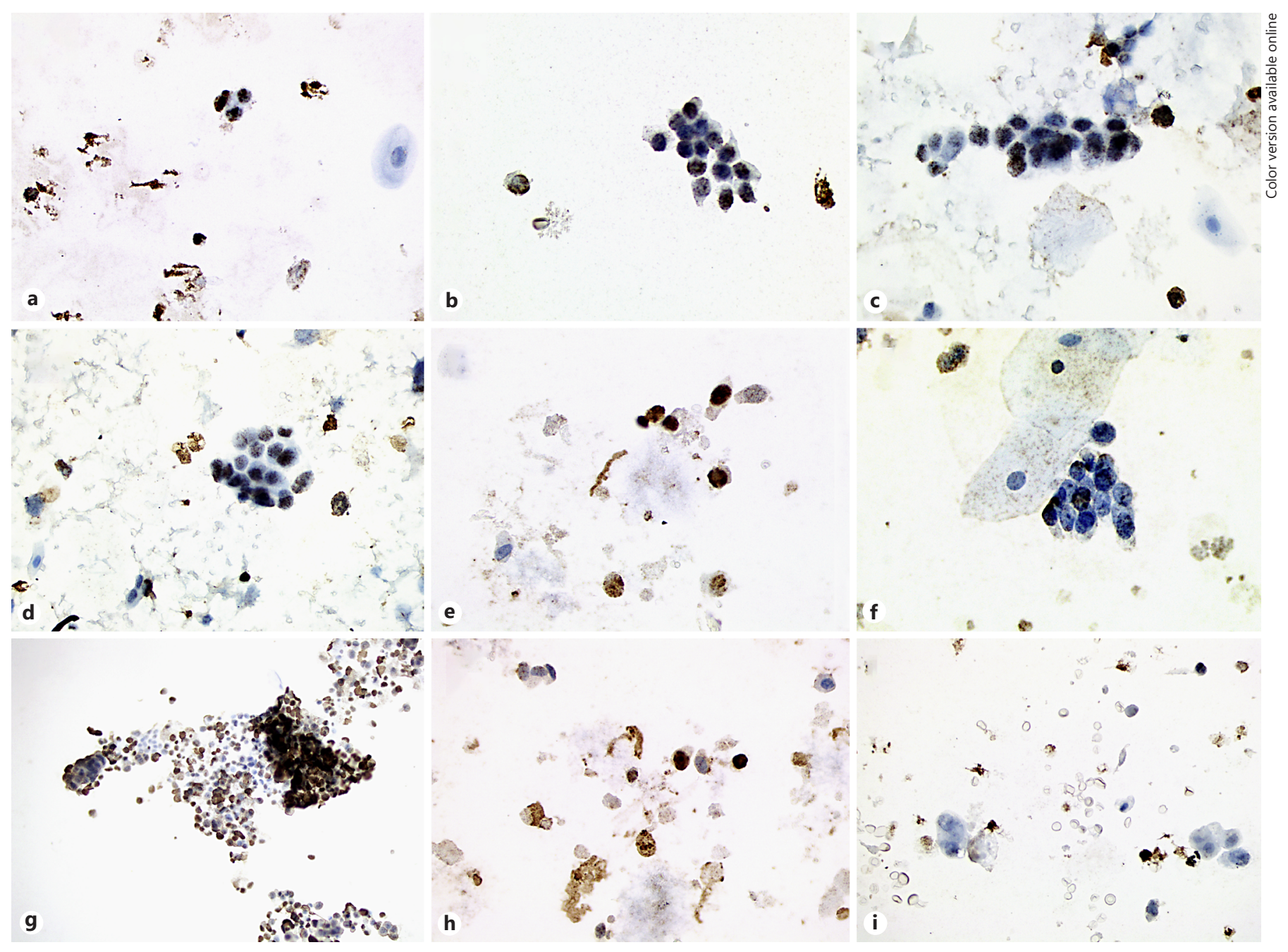

Fig. 6. Examples of hTERT staining. a A cluster of 3 cells (top center) stains positive; note the negative cell on the right side of the field. The corresponding diagnostic specimen was called "negative for urothelial atypia or malignancy"; the patient was subsequently found to have a low-grade urothelial neoplasm. $\mathbf{b}$ A cluster of atypical urothelial cells, the majority of which stain positive. The cor-

responding diagnostic specimen was called "atypical urothelial cells of uncertain significance"; the patient was subsequently found to have high-grade urothelial carcinoma. c-h Additional specimens in which hTERT was interpreted to be positive. i An example of a specimen containing high-grade urothelial carcinoma cells in which hTERT was negative.

An additional limitation of hTERT ICC is that nonurothelial cells may be positive, or are expected to be positive, and this may result in either an obscured field or misinterpretation of the cells as urothelial cells (false-positive result). hTERT ICC positivity is consistently observed in bacteria, neutrophils, and lymphocytes. This positivity, however, may be beneficial as it provides positive internal controls. The interpretation of hTERT ICC differs from most clinically utilized antibodies and likely requires experienced interpretation (Fig. 6). Other pitfalls are similar to those seen in slide-based, ancillary assays.

For instance, the cellular material present on the hTERT ICC slide may not be representative of that present on the slide created for clinical diagnosis; an atypical or suspicious diagnosis may be rendered due to rare, atypical cells; if these cells are not also present on the slide created for ICC, the ICC result would be noncontributory.

In evaluating the hTERT ICC, we have modeled how it may provide useful information when used in conjunction with cytology. The test may be most helpful in circumstances where a patient has a low-risk indeterminate UTC diagnosis (AUC-US) and a negative or absent cys- 
toscopy result. A positive hTERT result may identify a small subset of patients with an increased risk of HGUC who may otherwise not be closely followed, while a negative hTERT ICC result is associated with a slight reduction in risk for HGUC.

\section{Acknowledgments}

The authors wish to thank Mr. Minesh Lalla for his excellent technical support and advice during this study and Dr. Dorothy Rosenthal for her expertise and advice during optimization studies that preceded the current study.

\section{Disclosure Statement}

No relevant conflicts of interest exist.

\section{Funding Sources}

The materials and laboratory technologist time required for this study were funded by a research grant awarded to the Johns Hopkins University School of Medicine by Sienna Cancer Diagnostics.

\section{References}

$\checkmark 1$ Heney NM, Ahmed S, Flanagan MJ, et al: Superficial bladder cancer: progression and recurrence. J Urol 1983;130:1083-1086.

-2 Avritscher EB, Cooksley CD, Grossman HB, et al: Clinical model of lifetime cost of treating bladder cancer and associated complications. Urology 2006;68:549-553.

- 3 VandenBussche CJ: A review of the Paris system for reporting urinary cytology. Cytopathology 2016;27:153-156.

4 Barkan GA, Wojcik EM, Nayar R, et al: The Paris system for reporting urinary cytology: the quest to develop a standardized terminology. Acta Cytol 2016;60:185-197.

5 McCroskey Z, Pambuccian SE, Kleitherms S, et al: Accuracy and interobserver variability of the cytologic diagnosis of low-grade urothelial carcinoma in instrumented urinary tract cytology specimens. Am J Clin Pathol 2015; 144:902-908.

6 Zhang ML, Rosenthal DL, VandenBussche CJ: The cytomorphological features of lowgrade urothelial neoplasms vary by specimen type. Cancer Cytopathol 2016;124:552-564.

7 Miyanaga N, Akaza H, Ishikawa S, et al: Clinical evaluation of nuclear matrix protein 22 (NMP22) in urine as a novel marker for urothelial cancer. Eur Urol 1997;31:163-168.

8 Bubendorf L, Grilli B, Sauter G, Mihatsch MJ, Gasser TC, Dalquen P: Multiprobe FISH for enhanced detection of bladder cancer in voided urine specimens and bladder washings. Am J Clin Pathol 2001;116:79-86.
-9 Shay JW, Bacchetti S: A survey of telomerase activity in human cancer. Eur J Cancer 1997; 33:787-791.

10 Chen CH, Chen RJ: Prevalence of telomerase activity in human cancer. J Formos Med Assoc 2011;110:275-289.

11 Lin Y, Miyamoto H, Fujinami K, et al: Telomerase activity in human bladder cancer. Clin Cancer Res 1996;2:929-932.

12 Yoshida K, Sugino T, Tahara H, et al: Telomerase activity in bladder carcinoma and its implication for noninvasive diagnosis by detection of exfoliated cancer cells in urine. Cancer 1997;79:362-369.

13 Kamata S, Kageyama Y, Yonese J, Oshima H: Significant telomere reduction in human superficial transitional cell carcinoma. Br J Urol 1996;78:704-708

14 Kinde I, Munari E, Faraj SF, et al: TERT promoter mutations occur early in urothelial neoplasia and are biomarkers of early disease and disease recurrence in urine. Cancer Res 2013;73:7162-7167.

15 Cowan M, Springer S, Nguyen D, et al: High prevalence of TERT promoter mutations in primary squamous cell carcinoma of the urinary bladder. Mod Pathol 2016;29:511-515.

$\checkmark 16$ Nguyen D, Taheri D, Springer S, et al: High prevalence of TERT promoter mutations in micropapillary urothelial carcinoma. Virchows Arch 2016;469:427-434.
17 Zheng X, Zhuge J, Bezerra SM, et al: High frequency of TERT promoter mutation in small cell carcinoma of bladder, but not in small cell carcinoma of other origins. J Hematol Oncol 2014;7:47.

18 Cowan ML, Springer S, Nguyen D, et al: Detection of TERT promoter mutations in primary adenocarcinoma of the urinary bladder. Hum Pathol 2016;53:8-13.

19 Khalbuss W, Goodison S: Immunohistochemical detection of hTERT in urothelial lesions: a potential adjunct to urine cytology. Cytojournal 2006;3:18

20 Rosenthal DL, Vandenbussche CJ, Burroughs $\mathrm{FH}$, Sathiyamoorthy S, Guan H, Owens C: The Johns Hopkins Hospital template for urologic cytology samples. I. Creating the template. Cancer Cytopathol 2013;121:1520.

21 VandenBussche CJ, Sathiyamoorthy S, Owens CL, Burroughs FH, Rosenthal DL, Guan $\mathrm{H}$ : The Johns Hopkins Hospital template for urologic cytology samples. II and III. Improving the predictability of indeterminate results in urinary cytologic samples: an outcomes and cytomorphologic study. Cancer Cytopathol 2013;121:21-28.

22 Owens CL, Vandenbussche CJ, Burroughs FH, Rosenthal DL: A review of reporting systems and terminology for urine cytology. Cancer Cytopathol 2013;121:9-14.

23 Sullivan PS, Chan JB, Levin MR, Rao J: Urine cytology and adjunct markers for detection and surveillance of bladder cancer. Am J Transl Res 2010;2:412-440. 